\title{
Mechanics of the Right Whale Mandible: Full Scale Testing and Finite Element Analysis
}

\author{
Igor Tsukrov $^{\mathrm{a}^{*}}$, Judson C. DeCew ${ }^{\mathrm{b}}$, Kenneth Baldwin ${ }^{\mathrm{b}}$, \\ Regina Campbell-Malone ${ }^{\mathrm{c}}$, Michael J. Moore ${ }^{\mathrm{C}}$ \\ ${ }^{a}$ Department of Mechanical Engineering, University of New Hampshire, Durham, NH 03824, USA \\ ${ }^{\mathrm{b}}$ Center for Ocean Engineering, University of New Hampshire, Durham, NH 03824, USA \\ ${ }^{\mathrm{C}}$ Woods Hole Oceanographic Institution, Woods Hole, MA 02543,USA
}

\begin{abstract}
In an effort to better understand the mechanics of ship-whale collision and to reduce the associated mortality of the critically endangered North Atlantic right whale, a comprehensive biomechanical study has been conducted by the Woods Hole Oceanographic Institution and the University of New Hampshire. The goal of the study is to develop a numerical modeling tool to predict the forces and stresses during impact and thereby the resulting mortality risk to whales from ship strikes.

Based on post-mortem examinations, jaw fracture was chosen as a fatal endpoint for the whales hit by a vessel. In this paper we investigate the overall mechanical behavior of a right whale mandible under transverse loading and develop a finite element analysis model of the bone. The equivalent elastic modulus of the cortical component of right whale mandible is found by comparing full-scale bending tests with the results of numerical modeling. The finite element model of the mandible can be used in conjunction with a vessel-whale collision event model to predict bone fracture for various ship strike scenarios.
\end{abstract}

Keywords: right whale, mandible, mechanical testing, finite element analysis

\section{Introduction}

The North Atlantic right whale, Eubalaena glacialis, is one of the most critically endangered whales in the world [1]. Anthropogenic mortality, including deaths resulting from vessel-whale collisions and entanglement in fishing gear, accounted for 27 (64.3\%) of the 42 right whales examined postmortem between 1970 and January 2008 [2, 3]. Of the 21 vessel-related deaths examined postmortem, 11 (52.4\%) resulted from sharp (propeller) trauma, and 9 (42.9\%) resulted from blunt contact with the hull of a vessel. (In one additional case, the vessel-whale collision was reported anecdotally but when the carcass was examined no trauma mechanism could be determined based on the observed injuries.) A thorough review of available necropsy reports for right whales examined between 1970 and Dec 2006 revealed common injuries seen in right whales killed by vessels. These included hemorrhage, broken bones (i.e. skulls, jaws and 
vertebrae) and disarticulated ribs [2]. Vessel-whale collisions are being extensively studied using various approaches including historical data processing and hydrodynamic analysis [4-7].

The U.S. government recently enacted speed restrictions for vessels traveling through right whale critical habitat in an effort to reduce the likelihood of fatal collisions [8]. These restrictions will expire in five years unless they are found to be effective at reducing vessel-related mortality and are thereby reauthorized.

The ultimate goal of an on-going collaborative project conducted by the Woods Hole Oceanographic Institution (WHOI) and the University of New Hampshire (UNH) is to evaluate the efficacy of speed restrictions in terms of reducing the force of impact and thereby mortality due to blunt ship strikes. This is done via the creation of a finite element analysis (FEA) model to predict damage to whale skeletal components produced by various collision scenarios. The biomechanical modeling effort involves several components which are investigated separately and then combined to quantify the mechanics of interaction between a whale and a vessel during collision. In particular, one has to decide what kind of trauma should be considered as critical for a whale, evaluate the intensity of forces sufficient to cause the observed trauma, and determine whether such forces are produced for a given collision scenario (vessel size and approach speed, hull geometry, relative positions of whale and vessel prior to impact).

A previous study identified mandibular fracture as a viable fatal endpoint upon which a ship strike model could be based for several reasons [9]. Firstly, a fractured mandible has been found in 33\% of blunt ship-strike cases examined postmortem since 1970. Secondly, with appropriate failure criteria, bone fracture can be modeled as a binary condition (i.e. failure, no failure). In addition, no fully healed jaw fractures have been found to date in right whales, making mandibular fracture a reasonable proxy for fatality. The simple geometry of this bone lends itself well to modeling. Also, the right whale mandible has relatively thin soft tissue protection (on the order of $20 \mathrm{~cm}$ total, whereas $20 \mathrm{~cm}$ of a highly compliant blubber layer along with an even thicker layer of skeletal muscle cover the rest of the skeletal elements). Finally, the length of the mandible of large whales, measuring $25-30 \%$ of the total body length, represents one of the longest single bones in any extant group and is a relatively large target in the vessel-whale collision [9].

In this paper we investigate the overall mechanical behavior of a right whale mandible under transverse loading and develop a finite element analysis model of the bone. By combining fullscale mechanical experiments on the intact bone with numerical simulations, we determine the effective elastic modulus of cortical bone which is needed to calculate strains and stresses in the mandible during a collision event. The information on strain and stress concentrations can then be used with the corresponding bone fracture criterion (see, for example, [10]) to predict damage imparted on the whale due to ship strike. The numerical model of the overall collision event is presented in [11]; the experimental program to determine local elastic stiffness and breaking strength of trabecular and cortical components of the right whale mandible is described in [9] and [12].

This paper is organized as follows. Section 2 describes the mandible analyzed in this study (Fig. 1). The full-scale experiment conducted to evaluate mechanical behavior of the bone under 
transverse loading is described in Section 3. Section 4 focuses on the development of a finite element model of the mandible. In Section 5, the FEA model is used in conjunction with the experimental results to determine the equivalent mechanical properties of the cortical bone needed for the collision event model. Section 6 contains discussion of the results and establishes correlation of this work to the concurrent research efforts that include more detailed understanding of mechanical and physical properties of the right whale mandible tissue on the millimeter scale, as well as a related FEA effort to model the entire vessel-whale collision event.

\section{Right whale mandible analyzed in this study}

Individual right whales can be identified based on patterns of thickened skin patches found naturally on their heads and other unique markings. The New England Aquarium maintains a photo-identification catalog and sighting record for identified individuals who are each assigned an ID number [13]. This number begins with Eg (the initials of the scientific name for right whales, Eubalaena glacialis) followed by a 4-digit number. The mandible analyzed in this study was taken from right whale Eg\# 1004, a mature female right whale that died as a result of a vessel-whale collision. The animal measured $16.0 \mathrm{~m}$ (52.49 feet) in total length and was at least 29 years old, according to the sighting record [13]. At the time of necropsy, the robust blubber coat and excellent skin condition indicated that the animal was in very good health prior to death and lacked obvious signs of chronic illness or starvation. Eg\# 1004 had also successfully reproduced at least five times and was pregnant with her sixth known offspring at the time of death.

After a necropsy was performed on the animal, the left and right halves of the mandible were retrieved and were frozen within days of the necropsy. These bones represent the freshest right whale jaw bones available for study, with no appreciable loss of water or oil prior to sample preparation. The mandibles were wrapped in thick plastic sheeting and were kept at $-20^{\circ} \mathrm{C}\left(-4^{\circ}\right.$ F). The right half of the mandible was selected for full-scale testing and numerical modeling (Fig. 1). The plastic sheeting was removed and the bone was wrapped several times over in thin clear plastic wrap and was stored below room temperature for one week during full-scale mechanical testing. During this time it was estimated that less than 4 ounces of fluid seeped from the entire bone. The bone was then re-wrapped in the plastic sheeting and was refrozen. The tested mandible was $399.5 \mathrm{~cm}(13.11 \mathrm{ft})$ straight length and $449.5 \mathrm{~cm}(14.75 \mathrm{ft})$ external curved length. It weighed $224 \mathrm{~kg}$ (493.8 lbs). 


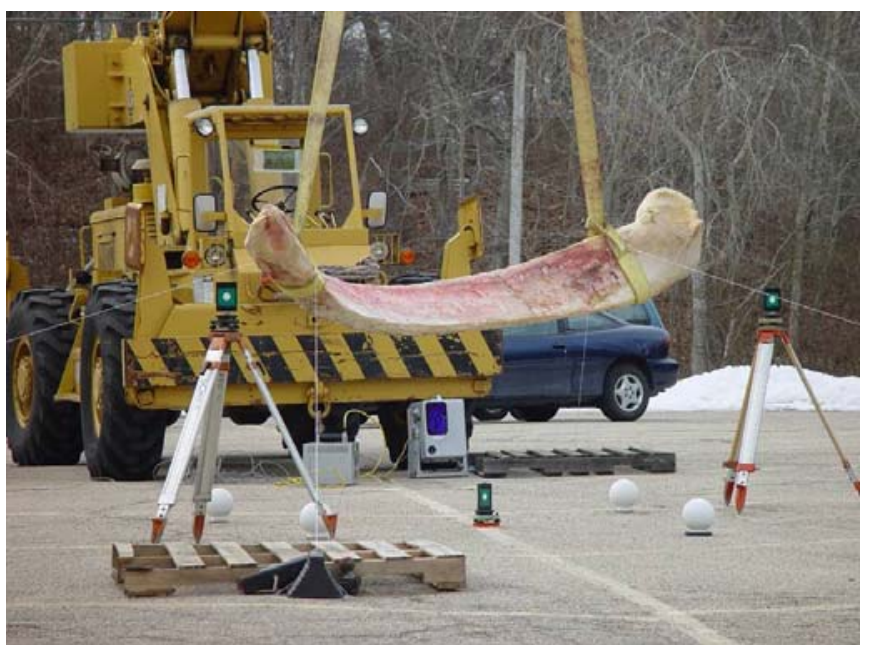

Figure 1: 3-D laser scanning of the mandible at the Woods Hole Oceanographic Institution (MA, USA) to determine the surface geometry.

\section{Full-scale mechanical testing}

The purpose of the full-scale testing was to evaluate the overall stiffness of the mandible when subjected to bending by transverse loading. The bone was loaded quasi-statically by a point force applied in the middle while two ends were simply supported. The strains and displacements in the designated locations were recorded for values of force varying from 0 to $4448 \mathrm{~N}$ (1000 lbf). These data can be used to determine the equivalent mechanical properties of the bone material, to validate predictions obtained using numerical simulations or simplified strength-of-material models, and to quantify deformation of the mandible during ship strike.

\subsection{Experimental set-up}

The choice of the experimental set-up was defined by three concerns. First, the loading and boundary conditions must provide deformation as close to pure bending as possible. This condition is not easily satisfied due to irregular cross-section of the bone and a complicated geometry of its ends. Secondly, the coordinate system must be clearly defined so that the experimental results could be compared with the numerical modeling predictions. And thirdly, the strain gage placement on the bone must be chosen to provide meaningful information on strain components during loading.

\section{$\underline{\text { Loading and boundary conditions }}$}

Due to the irregular cross-section of the bone and its inherent curvature it was decided that the best way to produce pure bending deformation was to suspend the bone from a gantry crane and load it by suspending weights (see Fig. 2 a). The bone was supported by two one-inch spectra straps on the left and right ends, and the weights were hung by two one-inch spectra strapping at the mid-point of the bone. This configuration provided the so-called "simple support" conditions free from the complications associated with constraining moments. This enabled greater ease when comparing the experimental tests on the bone to the numerical simulations as all the forces were in a single plane. 


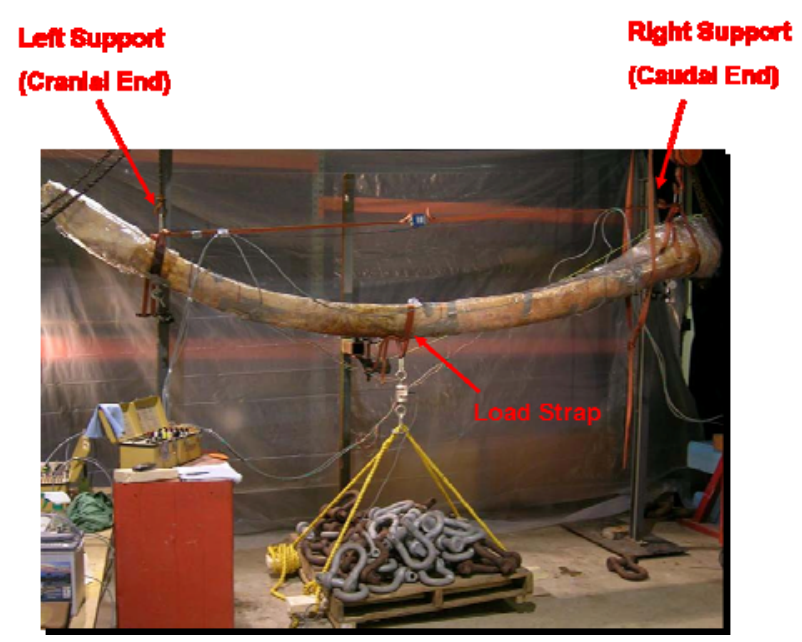

(a)

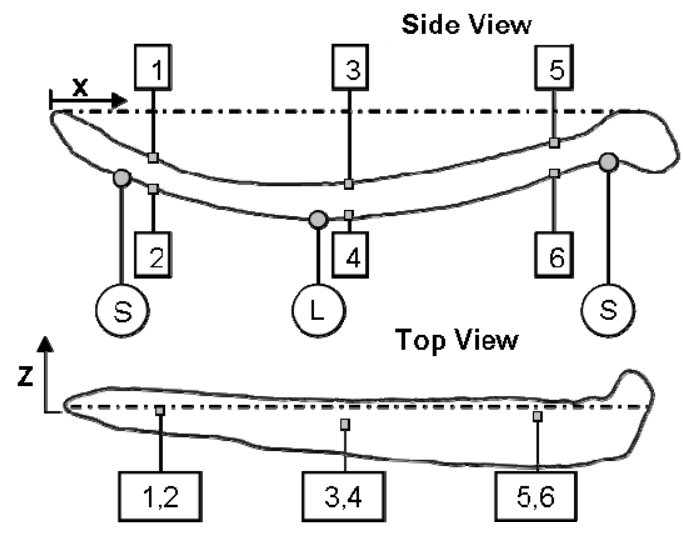

(b)

Figure 2 (a) Experimental set-up for full scale mechanical testing of the mandible. The "top" of the bone corresponds to the medial (lingual) surface of the right mandible while the "bottom" corresponds to the lateral (buccal) surface of the bone. (b) Schematics of the support and strain gauges locations.

\section{$\underline{\text { Coordinate system }}$}

A precise coordinate system was needed to relate the strains and deflections of the mandible to the finite element model. The longitudinal surface of the bone was denoted as the $\mathrm{X}-\mathrm{Z}$ plane, where the Y-direction was chosen to be vertical. Two endpoints, located at each end of the mandible, were used to center the $\mathrm{X}$-axis. Using this as a reference, the locations of each strain gauge, load application and support straps were recorded. These locations would then be investigated in the numerical model. The layout and coordinates of the straps and strain gages on the mandible can be seen in Fig. 2b.

\section{$\underline{\text { Strain gages }}$}

One strain gage rosette with gages at 0,45 , and 90 degree and a second, redundant, axial gage running in the X direction was placed at 6 locations. Sites 1, 3, and 5 are "on top" of the bone, while sites 2, 4, and 6 are "underneath" (see Fig. 2b). This should allow for compression and tension strains to be observed. The coordinates of the gage application points as well as locations of the attachment and loading straps are given in Table 1.

To facilitate mounting the gages to the mandible, the jawbone was thawed for two days. Plastic, wrapping the bone for the length of the test, was removed in the areas for gage attachment (see Fig. 3). After the gages were secured to the bone using M-Bond 200 (http://www.vishay.com), they were covered in wax to protect the strain gage, terminal, and wires from any unwanted contact and moisture. Three P3500 Strain Indicators and three Switch and Balance units were used to monitor the static strains during incremental loading. 


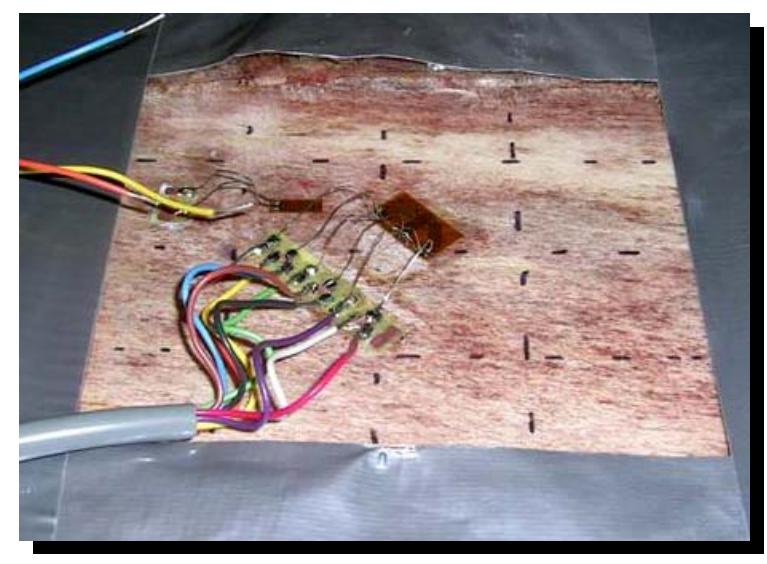

Figure 3. Typical area prepared for the application of the strain gages. The large central rosette is visible with a small strain gage seen off to the left.

Table 1. Location of the strain gages and strap attachment points.

\begin{tabular}{ccc}
\hline Site & $\begin{array}{c}\text { X Axis } \\
(\mathrm{cm})\end{array}$ & $\begin{array}{c}\text { Z Axis } \\
(\mathrm{cm})\end{array}$ \\
\hline \hline 1 & 90.8 & 10.8 \\
2 & 90.8 & 10.8 \\
3 & 196.8 & 16.5 \\
4 & 196.8 & 16.5 \\
5 & 276.2 & 11.4 \\
6 & 276.2 & 11.4 \\
Left Strap & 54.6 & 12.7 \\
Load Strap & 177.8 & 15.2 \\
Right Strap & 354.3 & 10.2 \\
\hline \hline
\end{tabular}

\subsection{Testing procedure}

The experimental configuration for the mandible is shown in Figure 2a. In the initial measurement, the strain gages were zeroed before the bone was suspended. This enabled the strains due to the bone weight to be assessed. The bone was then hung from the testing frame, and the strain gages were read and recorded. Three sets of measurements were conducted. In the first set, the strain measurements were read and recorded in load increments of approximately $444 \mathrm{~N}$. The small load increment resulted in copious data, but required keeping careful records of the actual weight. A $22.2 \mathrm{kN}(5000 \mathrm{lb})$ load cell was placed between the pallet with the weights and the bone to determine the exact amount of weight applied to the bone. After every $444 \mathrm{~N}$ increment was applied, the weight was taken off and the zeroes read again. This procedure was used to account for signal drift and possible seepage of fluids through the bone due to handling. The process continued until a desired weight of $4448 \mathrm{~N}$ (1000 lbs) was achieved. The second and third tests were simplified slightly. Weights were incremented by $890 \mathrm{~N}$ for every reading up to a final weight of $4448 \mathrm{~N}$. 


\subsection{Testing results}

Figure 4 presents values of strains measured at six locations on the surface of the bone (see Table 1) as a function of the applied load. Solid lines in the figure indicate the linear trends of the corresponding data sets with the constraint that strain is zero when no external load is applied. Note that the data acquisition procedure described in section 3.2 allows separation of the deformation due to loading (presented in Fig. 4) from the deformation caused by self-weight.

For the purpose of compactness and to make it easier to compare the magnitudes of strains in different directions at the same location, the plots present the absolute values of the observed strain. The data sets corresponding to negative strain (compression) are denoted by “(-)“ sign in the graph legends. Also, more data points were available for longitudinal strain due to redundant strain gages used in this direction of interest.

It can be seen that locations 5 and 6 exhibit much higher variance of measured values as compared to locations 1-4 (Fig. 2b). The coefficients of determination, $r^{2}$, for the longitudinal strain were found to range from 0.78 (location 6 ) to 0.99 (location 3). This can be explained by the geometrically complex three-dimensional shape of the caudal end of the bone. The area of contact between the bone and the supporting strap in these locations has irregular shape so that minor shifts and readjustments of the straps during loading produced noticeable variations in the recorded strain. However, the coefficients of determination indicate that the linear trends are still well pronounced, especially, in longitudinal direction. 

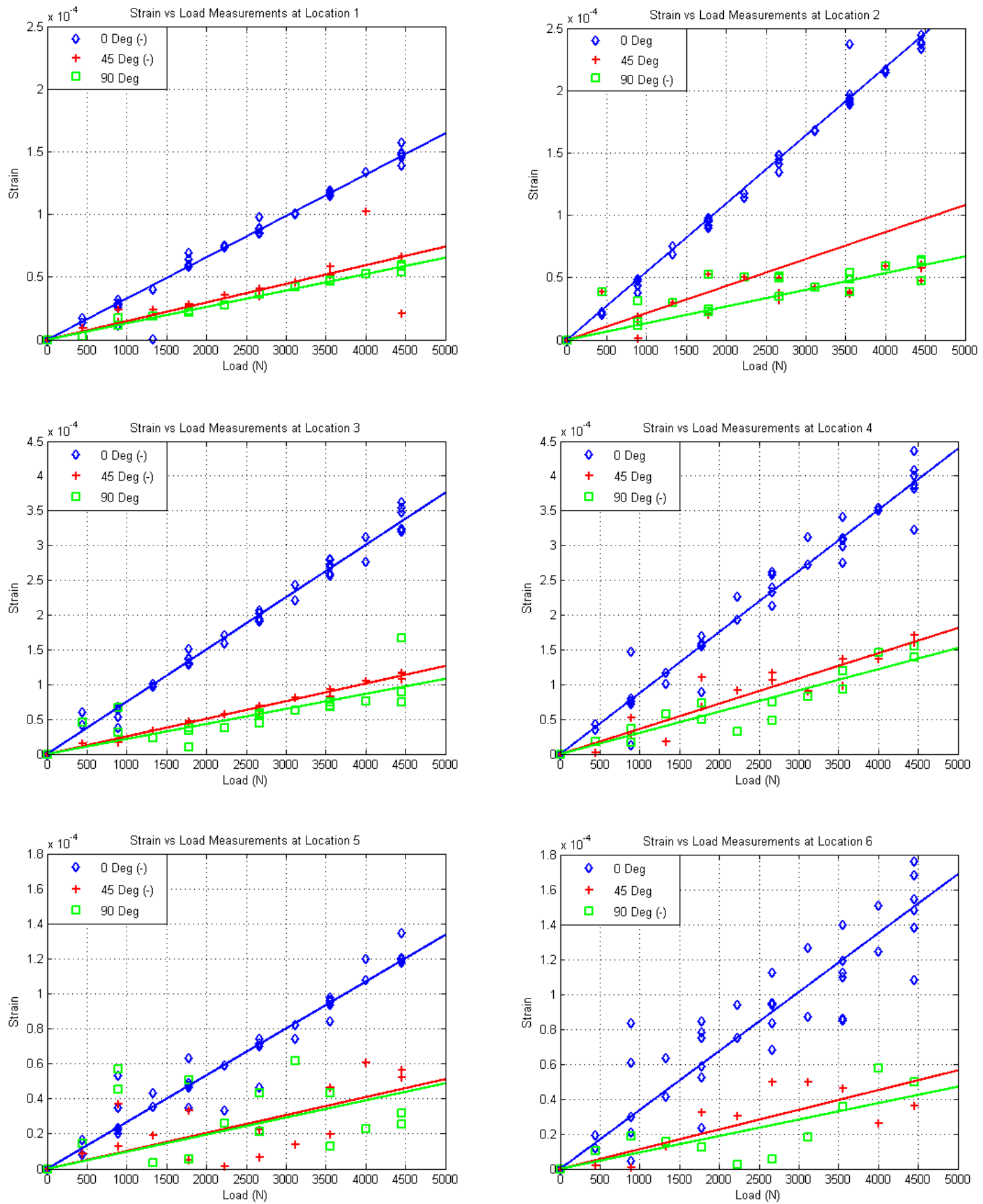

Figure 4. Strains in longitudinal $\left(0^{\circ}\right)$, inclined $\left(45^{\circ}\right)$ and transverse $\left(90^{\circ}\right)$ directions. Compressive strains are denoted by (-) in the graph legends. Solid lines are linear trends of the corresponding data sets. 


\section{Numerical model}

A three-dimensional numerical model of the bone was developed (1) to understand the deformation of the bone under transverse loading, and (2) to be used in numerical simulations of the vessel-whale collision event. The model utilizes the finite element analysis (FEA) method as described, for example, in Bathe [14]. FEA has been successfully used recently to analyze threedimensional deformation of bones [15-17] and the human mandible in particular [18]. The shape of the right whale mandible was modeled utilizing data from a three-dimensional laser scan of the same mandible that was subjected to full-scale mechanical testing. The material properties of the bone can be assigned either based on the density distribution, or as a trabecular core /cortical shell material system. By reproducing the full-scale tests presented in Section 3, the model was utilized to determine the equivalent (homogenized) elastic modulus of the cortical bone that can be used in the ship strike numerical modeling.

\subsection{Development of finite element mesh}

The first step in the numerical analysis of the mandible was to construct a geometric model based on the digital information on the bone geometry. The mandible described in Section 2 was laser scanned using the Cyrax 2500 Laser Scanning System provided by Meridian Associates (Beverly, MA, http://www.meridianassoc.com/contact.htm) to acquire the coordinates of the bone surfaces (Fig. 1).

The obtained data consisted of 38,725 sets of $\mathrm{X}, \mathrm{Y}$, and $\mathrm{Z}$ coordinates of points from measurements around the entire surface of the mandible. When viewed in a standard CAD program, the surface of the mandible could be seen as a "cloud" of individual points (see Fig. 5). These data points needed to be transformed into a continuous surface for finite element analysis. To perform this task, the software package Rhinoceros ${ }^{\circledR}$ (http://www.rhino3d.com) was utilized. This program has the ability to manipulate point clouds, surfaces, polygon meshes, etc. for complex geometries and utilizes Non-Uniform Rational B-Splines (NURBS) to construct curves and surfaces.

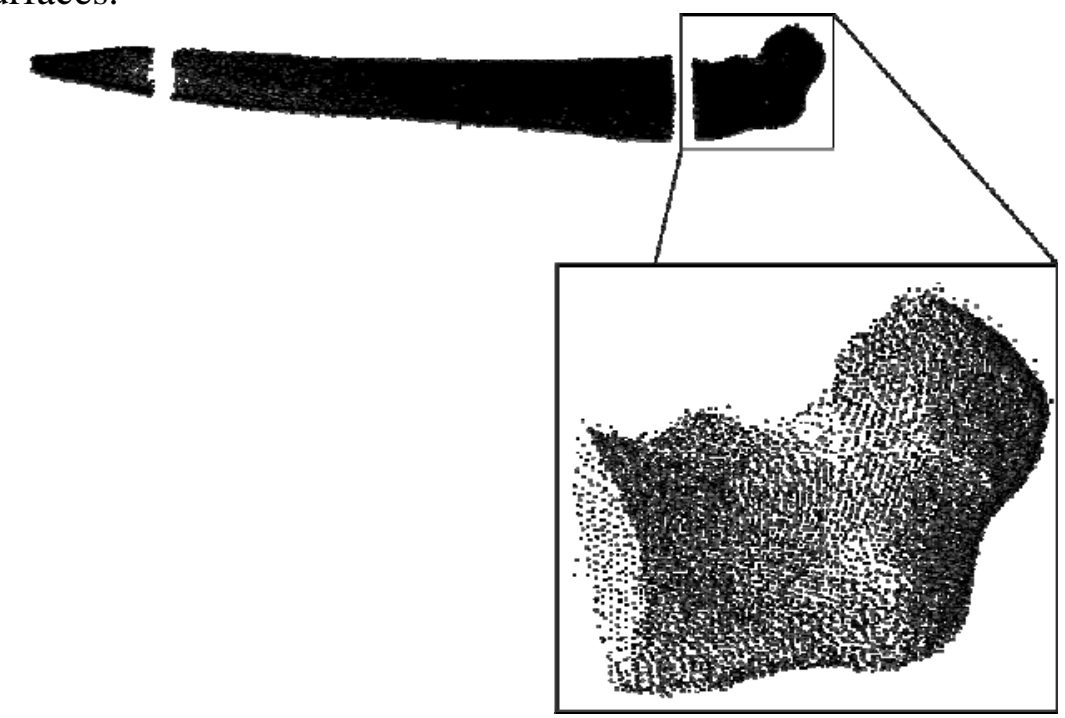

Figure 5: The laser scanned data points representing the mandible surface. Point coordinates can be used to generate a surface representing the jaw bone. 
Generating a mandible surface from the data presented several challenges due to the size and complex shape of the point cloud. Duplicate points and measurement error caused by the utilized scanning instruments added points "off" the surface and skewed surface generation (Fig. 5). Therefore, the data points were cleaned up and organized for use in Rhino. The first step was to sort the data along the main axis of the bone. Duplicate data points were then eliminated. To generate the jawbone surface, cross-sections or "slices" of the laser scanned individual points were obtained (see Fig. 6). These sections were taken in $5 \mathrm{~cm}$ (2 inch) intervals on portions where the bone had a complex geometry, such as the caudal end (ramus) and the cranial end ("chin"). In areas where resolution was not as critical, such as the interior length, the spacing between cross sections was increased to $30 \mathrm{~cm}$ (12 inches). A curve was then generated for each "slice" forming an outline of the cross-section (shown on Fig. 6a). These curves were extrapolated to form the outer surface of the mandible (Figs. 6b and 6c).

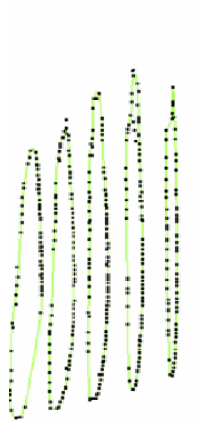

(a)

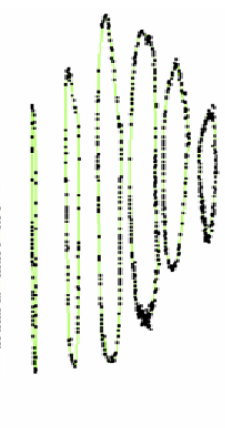

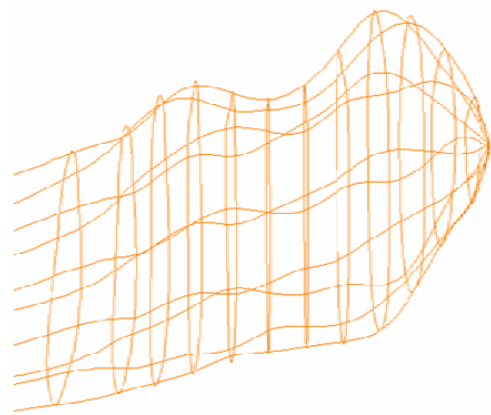

(b)

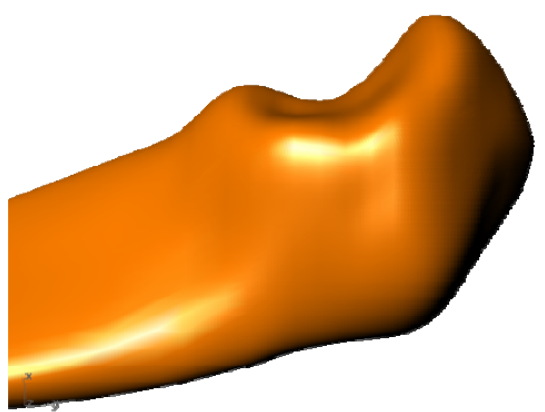

(c)

Figure 6: The surface generation of the ramus portion of the mandible. Cross sections of data points were taken at set intervals and curves were formed through these points (a). A skin and subsequent surface was formed based on the cross sections (b) and (c).

To perform FEA, the geometric model of the bone surface was exported into MSC.MARC, a general purpose FEA software package (http://www.mscsoftware.com). This surface model was used to develop the three-dimensional finite element mesh of the bone. Several iterations of the automated mesh generation procedure were performed to optimize the mesh while making sure that all details of the complex surface geometry of the mandible are captured. The final mesh contained 18290 hexagonal elements with 25239 nodes as can be seen in Fig. 7.

Figure 8 shows a cross-sectional slice of the mesh. Several such cross-sections were analyzed to evaluate the thickness of the external layer of finite elements. This is important in the material property assignment discussed in the next section. Although difficult to determine for every element (due to the non-uniformity of the mesh generation), the thicknesses (into the bone) of 10 randomly selected exterior elements were measured. They were found to be in the range from 1.05 to $2.12 \mathrm{~cm}$, with typical values of approximately 1.5 to $1.75 \mathrm{~cm}$. 


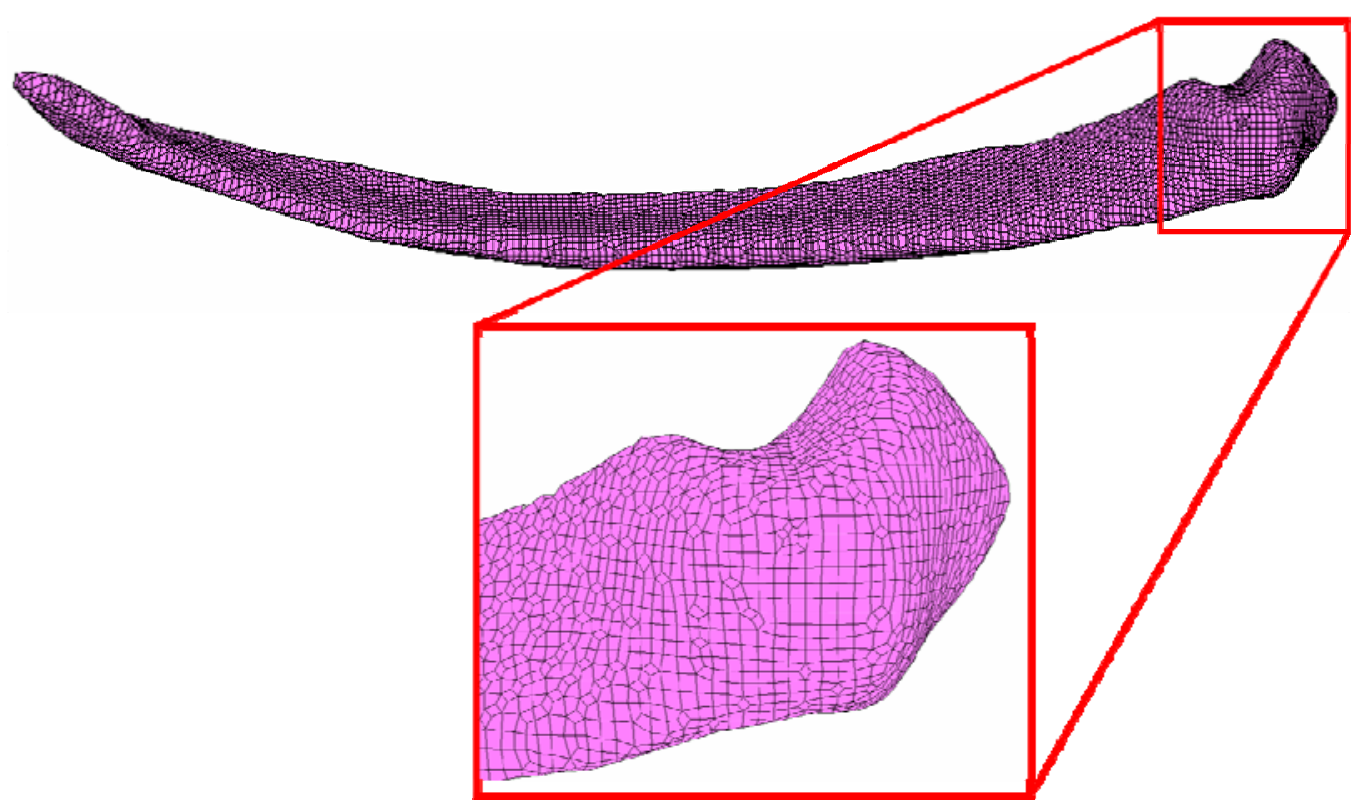

Figure 7: The general purpose FEA package produced a three dimensional mesh with 18290 elements.

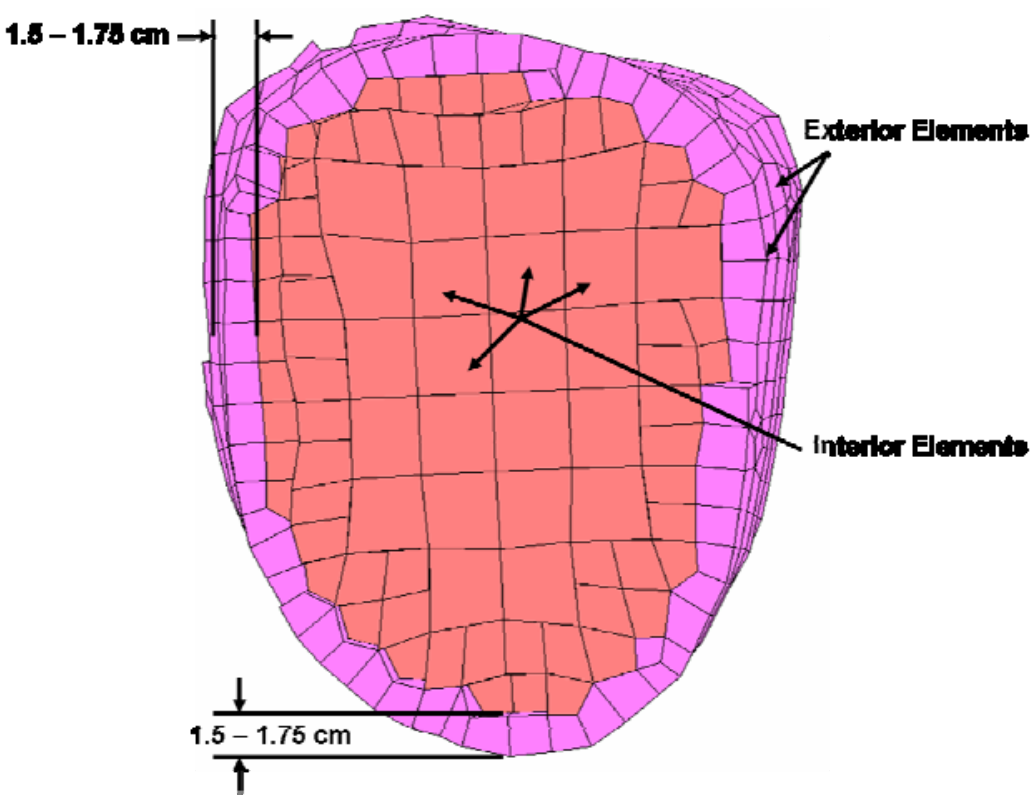

Figure 8: The average exterior element depth ranged from 1.5 to $1.75 \mathrm{~cm}$. The interior elements were up to 4 times larger.

\subsection{Assignment of the material properties}

Once the finite element mesh was generated, the material properties could be assigned. Computed tomography scans of the right whale mandible confirm that it has an internal microstructure similar to other bones. It consists of a thin outer shell of dense cortical bone and a core (central region) of porous trabecular bone. The structure and material density of these two 
different bone types result in differences in their material behavior. In addition, mechanical properties of the mandible are noticeably inhomogeneous within each bone type (vary widely throughout in both longitudinal and transverse directions) and exhibit substantial inter-specific differences [12, 19-22]. In order to provide an accurate representation of the jawbone material, two geometrically identical FEA models were created with different material property assumptions. The "shell" model was simplified to have two materials representing the outer cortical bone and inner trabecular core. The "density based" model allowed for assignment of the mechanical properties based on the value of material density at a given point of the bone.

\section{“Shell” Model}

The "shell" model was constructed such that the interior and exterior elements were assigned to two different material groups (Fig. 9). Due to the model’s size (18290 elements) manual selection of the elements for material property assignment was not practical. Therefore, a supplementary program was written in an engineering computation software package, MATLAB (http://www.mathworks.com), utilizing the laser scan measurements. Recall that the mandible solid model was generated from laser scan data (X, Y, Z locations). To assign the material properties, the trabecular bone modulus of elasticity and Poisson's ratio values were initially assigned to all elements. The laser-scanned points (with associated material properties) were then compared to the geometric center of each element in the FEA mesh. The program determined if the laser scan location was within $1.5 \mathrm{~cm}$ and if so, assigned the cortical bone properties to that element. If the program did not find a scan measurement within the required distance, the properties were not re-assigned. However some exterior elements were skipped because there was not any scan measurements within the required distance. These remaining elements were manually reassigned their correct material properties.

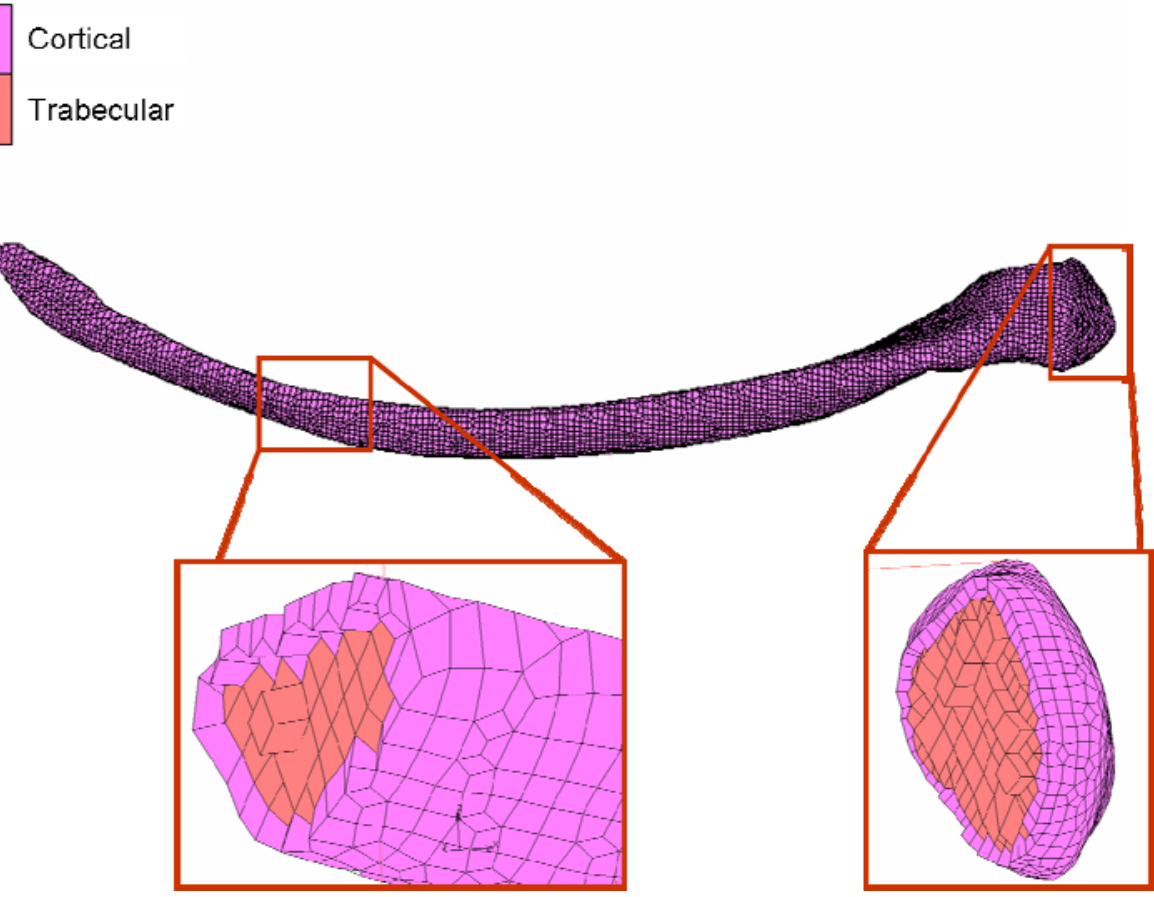

Figure 9: The cortical and trabecular bone properties assigned in the "shell" model 
The material properties of the trabecular bone were determined using experiments conducted on 76 specimens taken from the mandible after the full-scale testing was performed. Cylindrical specimens each measuring approximately $7.5 \mathrm{~mm}$ in diameter and $10 \mathrm{~mm}$ in height were manufactured and subjected to uniaxial compression tests. The details on the testing procedure and data processing techniques used are presented in [9, 12]. Based on the experimental results the values of $E_{\mathrm{T}}=357.2 \mathrm{MPa}$ and $v_{\mathrm{T}}=0.06$ were chosen for the FEA model. The selection of mechanical parameters for cortical bone is discussed in Section 5.

\section{Model based on density}

A second FEA model implemented assignment of elastic properties based on the local values of material density. It has been observed in the literature that Young's modulus and compressive strength of the bone exhibit a positive correlation with density [23-27]. Different definitions of density are used in bone biomechanics literature to correlate with material properties. Quantitative computer tomography (QCT) density used in this study is the density of hydrated bone containing intertrabecular fat as measured using calibrated computed tomography. Note that this measure is different from the apparent density which is defined as the mass of hydrated, defatted bone divided by the geometric volume of the specimen. The advantages of mapping the density of such a large structure using QCT were substantial - allowing the internal density to be mapped non-destructively and on a fine scale. Additionally, destructive sampling was carried out on a small portion of the bone [12] such that apparent density measures and mechanical testing could be carried out on a subset of the same bone, permitting correlation with published biomechanics data.

The mandible was computed tomography (CT) scanned on a Siemens Somatom Volume Zoom Spiral CT scanner (Munich, Germany, http://www.medical.siemens.com). These scans were conducted using both soft tissue (H40) and bone (H70) protocols allowing x-ray beams to pass through the sample, where they are altered (attenuated) and picked up on the other side of the sample by four rows of detectors. Each bone was scanned at $3 \mathrm{~mm}$ increments to ensure that three-dimensional data points, known as attenuation coefficients, were gathered from a significant portion of the bone sample. The attenuation coefficients were compared to the coefficients of six hydroxyapatite standards (Computerized Imaging Reference Systems, Norfolk, VA, www.cirsinc.com) ranging in density from 100 to 1250 milligrams per cubic centimeter (mg/cc). Using these standards the attenuation coefficients (expressed in Hounsfield units) were converted into apparent density (mg/cc) values.

The next step was to assign these values to the respective finite elements. To accomplish this task, a supplementary program was written in MATLAB. Similarly to the procedure employed for the "shell" model, the program compared the location of the CT scanned data points with the center of each element. If the distance was within $3 \mathrm{~cm}$, the corresponding material properties were assigned. If there were no CT measurements within $3 \mathrm{~cm}$, a default material property (either cortical or trabecular) was assigned.

In an effort to better visualize and simplify the model, the densities values were grouped together in ranges. These ranges allowed for full visualization of the applied materials. The cortical and 
trabecular densities were each grouped into 10 ranges, shown in Table 2 . The average range value was then assigned to all elements within the range. (Note that the average range value was utilized, not the average density value within a range.) This format resulted in 22 different material properties (10 cortical, 10 trabecular, 1 cortical default, 1 trabecular default). The results of the material property application can be seen in Fig. 10.

Table 2: The grouped QCT density values assigned to the FEA mesh

\begin{tabular}{cccc}
\hline \hline \multicolumn{2}{c}{ Cortical Bone } & \multicolumn{2}{c}{ Trabecular Bone } \\
$\begin{array}{c}\text { QCT Density } \\
\text { Range } \\
(\mathrm{g} / \mathrm{cc})\end{array}$ & Assigned Value & $\begin{array}{c}\text { QCT Density } \\
\text { Range }\end{array}$ & $\begin{array}{c}\text { Assigned Value } \\
(\mathrm{mg} / \mathrm{cc})\end{array}$ \\
\hline \hline $0.090-0.214$ & 0.152 & $0.020-0.069$ & 0.0445 \\
$0.214-0.338$ & 0.276 & $0.069-0.118$ & 0.0935 \\
$0.338-0.462$ & 0.400 & $0.118-0.167$ & 0.1425 \\
$0.462-0.586$ & 0.524 & $0.167-0.216$ & 0.1915 \\
$0.586-0.710$ & 0.648 & $0.216-0.265$ & 0.2405 \\
$0.710-0.834$ & 0.772 & $0.265-0.314$ & 0.2895 \\
$0.834-0.958$ & 0.896 & $0.314-0.363$ & 0.3385 \\
$0.958-1.082$ & 1.02 & $0.363-0.412$ & 0.3875 \\
$1.082-1.206$ & 1.144 & $0.412-0.461$ & 0.4365 \\
$1.206-1.330$ & 1.268 & $0.461-0.510$ & 0.4855 \\
Default & 1.123 & Default & 0.152 \\
\hline \hline
\end{tabular}

Trabecular_1

Trabecular_2

Trabecular_3

Cortical_1

Trabecular_4

Trabecular_5

Cortical_2

Trabecular_6

Trabecular_7

Trabecular_8

Cortical_3

Trabecular_9

Trabecular_10

Cortical_4

Cortical_5

Cortical_6

Cortical_?

Cortical_8

Cortical_9

Cortical_10

Trabecular_default

Cortical_default

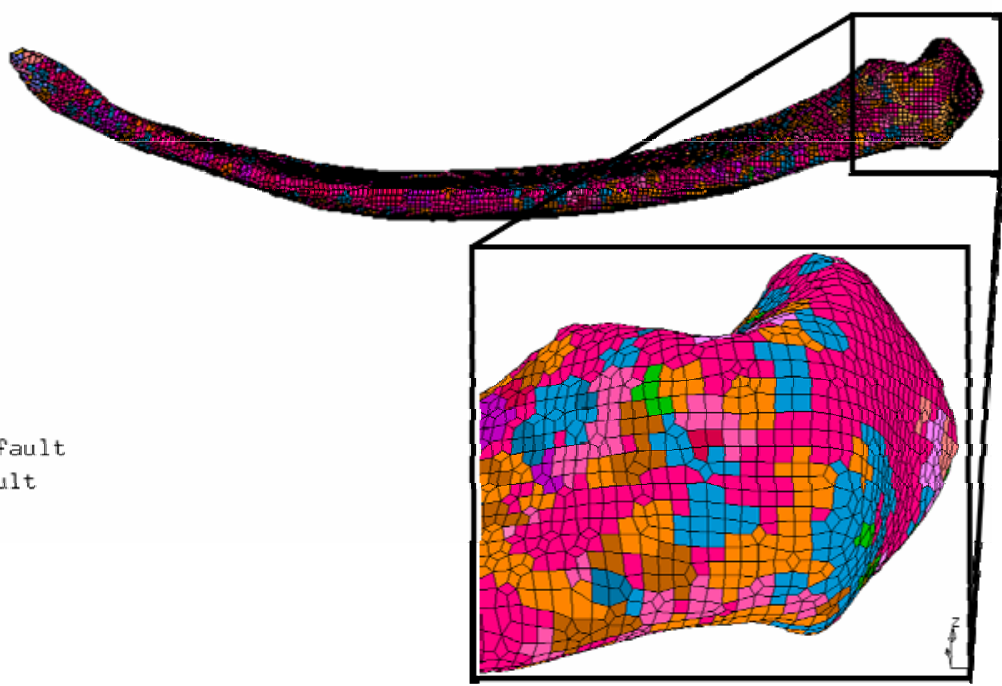

Figure 10: The mandible with 22 applied material properties 
It is important to mention that similar to the well-studied human bone, the right whale mandible has a thin "shell" (cortex) composed of dense cortical bone surrounding a core of porous, low density trabecular bone tissue. Both cortical and trabecular bone are defined based on their unique microstructure and relative densities - although there is some overlap between low density cortical and high density trabecular bone as seen in Table 2. In a typical mammal, however, there is a distinct transition from cortical to trabecular bone tissue as one moves from the periosteum (outer surface of the bone) toward the core. It was recently discovered that the transition between the cortical cortex and the trabecular core of the right whale mandible is gradual, resulting in a noticeable region of "intermediate" bone with intermediate microstructure and density [9].

Presently, there is a paucity of available data to establish a reliable correlation between density and elastic modulus for the entire range of trabecular and cortical bone properties of the right whale mandible. In fact, the presence of the "intermediate" bone requires development of special quantitative relationships for the whale bone. Hence, for the purpose of development of vessel whale collision model, the simplified "shell” model approach to determine the material properties of the mandible is presently used.

\section{Extraction of equivalent mechanical properties}

Mechanical and physical properties of right whale mandible are inhomogeneous and anisotropic $[9,12]$. They exhibit significant variations in both cross-sectional and longitudinal directions. Modeling of strain gradients and stress concentrations produced by such variations is outside of the scope of this paper. We are concerned with the overall mechanics of bone and, in particular, its deformation under transverse loading.

Full scale mechanical testing reported in Section 3 confirms that for the considered range of loading, the assumption of linear elastic behavior is appropriate. Our numerical simulations utilize the simplified "shell" model to define the mechanical properties of the bone, as discussed in Section 4. In this model, we assume that the overall response can be predicted by considering two linearly-elastic isotropic constituents of the bone, cortical and trabecular, characterized by their corresponding Young's moduli $E_{C}, E_{T}$ and Poisson's ratios $v_{C}, v_{T}$. Our preliminary studies indicate [28] that out of these four parameters the elastic modulus of the cortical bone $E_{C}$ plays the key role. However, not enough information is presently available to make a reliable prediction for this parameter based either on bone microstructure or a sufficient number of millimeter-scale tests / measurements. Thus, the full scale testing and FEA simulations were combined to estimate the macroscopic, or "equivalent”, value of $E_{C}$ for the right whale jaw bone in bending.

Numerical simulations were performed to reproduce the physical testing described in Section 3. The boundary and loading conditions were chosen to replicate the full scale test; the load of up to $4448 \mathrm{~N}$ (1000 lbf) was considered. The FEA model was aligned to a coordinate system similar to the one used in laboratory measurements. The main axis of the jawbone was set-up in the X- 
direction, as shown in Fig. 11. Using data presented in Table 1, the orientation of the jawbone, supports and load placement were reproduced. The vertical lines in the FEA model represent the strain measurement locations.

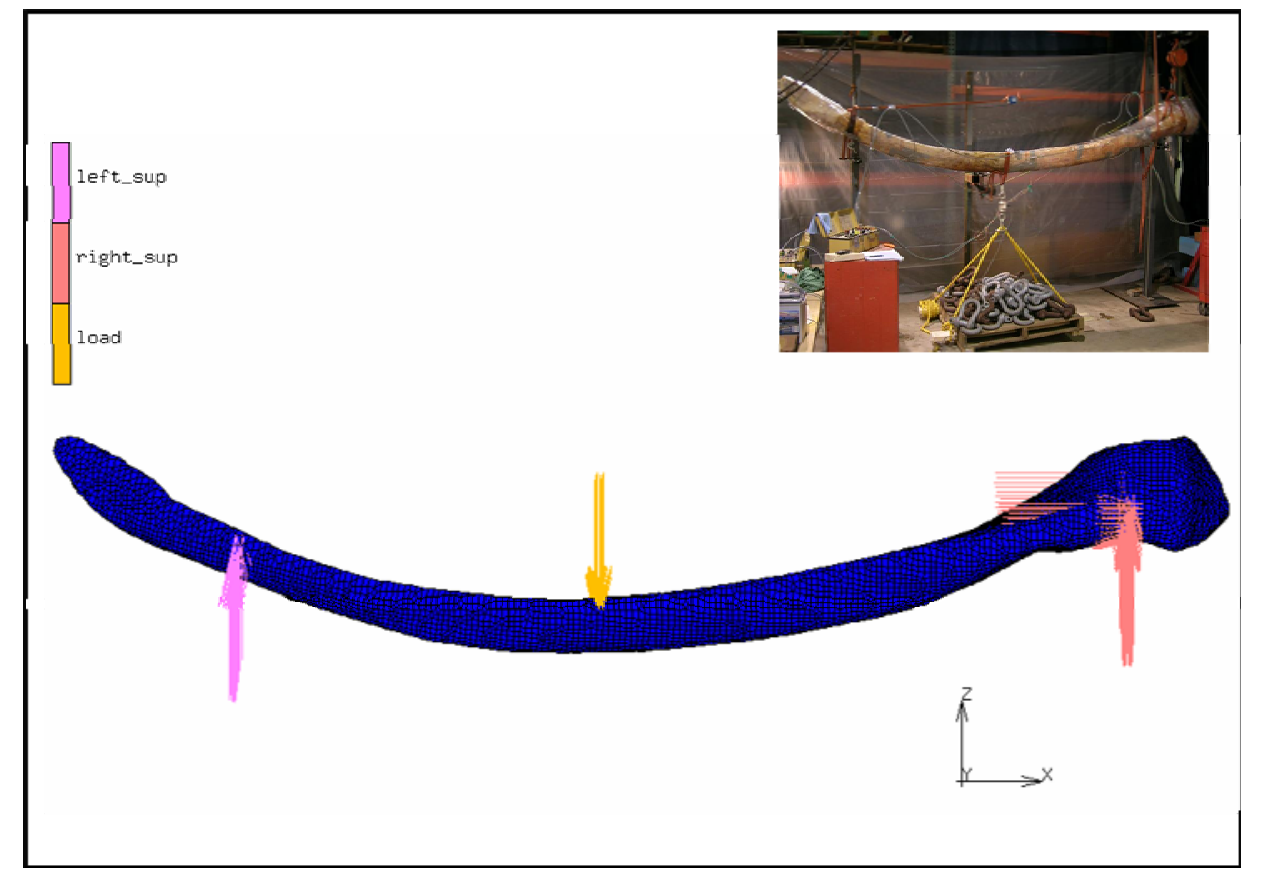

Figure 11: The FEA model was placed in a similar orientation and boundary conditions as the laboratory experiments.

The material properties of the trabecular bone were chosen based on the set of tests conducted on 76 cylindrical specimens as described in section 4.2 The values of the Poisson's ratio for the cortical bone reported in the literature vary from 0.12 to 0.63 , see $[19,29,30]$ and discussion in Shahar et al. [31]. Our preliminary simulations did not show any substantial sensitivity of the predicted bending response on this parameter across the reasonable range of values [28], similarly to the observations reported in Yosibash et al. [15], so the value $v_{C}=0.3$ was used.

The FEA predicted longitudinal strain at locations 3 and 4 was examined and compared to the measured values in order to choose the equivalent Young's modulus of the cortical bone. The average value was determined as follows

$$
E_{c}=\frac{1}{2}\left(E_{3}+E_{4}\right)
$$

where $E_{i}$ is the value of the elastic modulus of the cortical bone chosen in the numerical model to match the measurements of the longitudinal strain at the location $i$. A series of simulations were performed and values $E_{3}=7.93 \mathrm{GPa}$ and $E_{4}=8.05 \mathrm{GPa}$ were obtained. Based on these values, the equivalent Young's modulus value of $E_{c}=7.99 \mathrm{GPa}$ was chosen. Fig. 12 provides load-strain graphs at locations 3 and 4 predicted using the equivalent value of $E_{c}$ as well as the 
data points obtained from the full-scale experiment. Good correspondence between numerically predicted and experimentally observed values of longitudinal strain can be seen throughout the entire range of deformation.
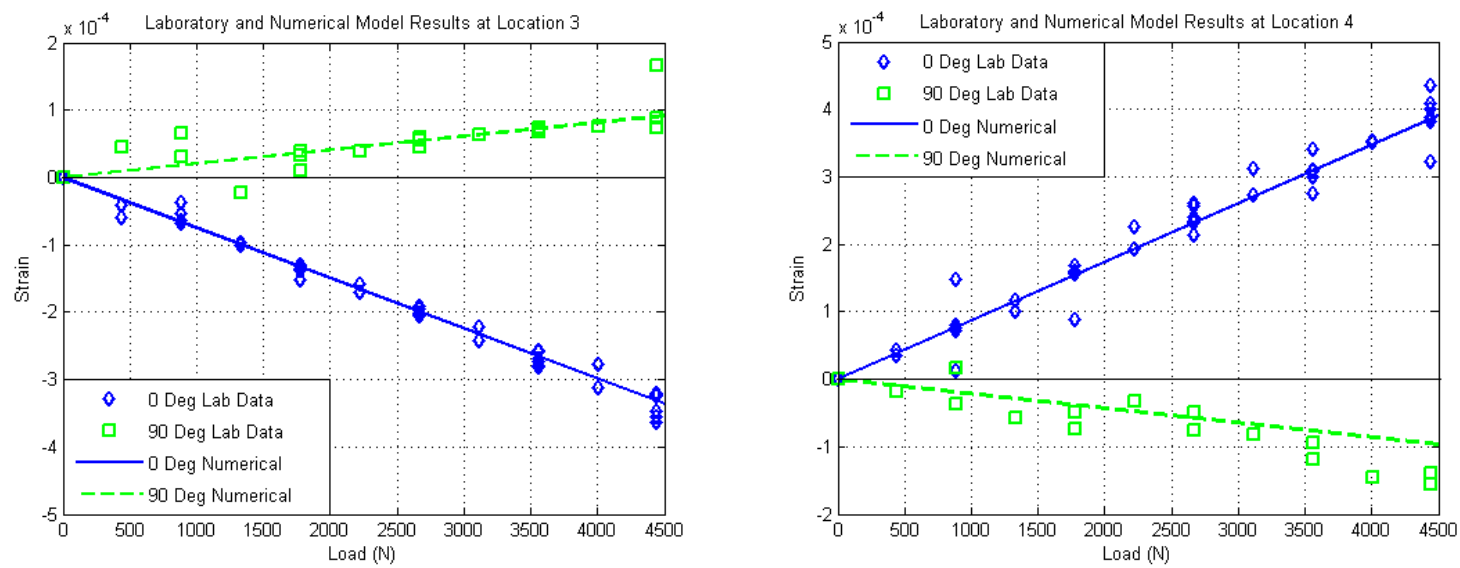

Figure 12: Comparison of the numerically predicted and experimentally observed strains at the "top" (location 3) and "bottom" (location 4) points in the middle cross-section of the bone.

Note that the value of $E_{c}$ determined using the above procedure results in significant discrepancies between the predicted and measured values of the transverse strain: $\delta=5 \%$ for location 3 and $\delta=15 \%$ for location 4 , where $\delta$ is defined as $\delta=\left|\varepsilon_{\text {tests }}-\varepsilon_{\text {fea }}\right| / \varepsilon_{\text {max }}$. This discrepancy can be expected because the anisotropic and inhomogeneous material properties of the bone tissue are represented by the equivalent elastically-isotropic material models. Thus, the proposed set of material parameters, while being appropriate to describe the overall bending of the bone, should not be used to model local stress concentrations in the right whale mandible under complex non-homogeneous loading conditions.

One of the popular approaches to study bone fractures during blunt impacts is to model bones as beams. Such an approach was successfully used, for example, by Chen [32] and Niu et al. [33] to study impact injuries of human ribs. However, direct application of such a simplified approach to analysis of bending of a whale mandible introduces significant inaccuracies in the predicted deformations. Consider, for example, the mandible studied in this paper loaded by point force of $4448 \mathrm{~N}$ (1000 lbf) in pure bending as shown in Fig. 2. If it is treated as a beam of uniform elliptical cross-section having the semi-axes corresponding to the dimensions at the position of load application ( $a=0.14 \mathrm{~m}, b=0.08 \mathrm{~m}, I_{y y}=5.64 \times 10^{-5} \mathrm{~m}^{4}$ ) and Young's modulus $E=7.99 \mathrm{GPa}$, the maximum value of the analytically predicted strain is $1.41 \times 10^{-3}$ which is approximately 3 times greater than the experimentally observed value of $0.44 \times 10^{-3}$ at location 4 . This discrepancy caused by approximation of the actual bone geometry by a simplified shape may have significant implications since many researchers consider strain to be the governing parameter of bone fracture [10, 34]. 


\section{Conclusions}

In this paper, full scaled mechanical testing was combined with finite element numerical simulations to investigate the overall mechanical behavior of the right whale mandible subjected to bending by transverse loading. Understanding and proper numerical modeling of mandible biomechanics is necessary to produce a predictive biomechanical model of a vessel-whale collision event. Such a model is presently being developed and can be used to evaluate the efficacy of various measures implemented to reduce whale mortality due to ship strike.

Full scale mechanical testing was conducted on a $224 \mathrm{~kg}$ mandible of a deceased right whale. The bone was suspended at two ends and loaded by centrally applied incrementally increasing force. The components of strain at six locations on the surface of the bone were recorded in a well defined coordinate system. Analysis of the results shows approximately linear mechanical response for the considered range of loading.

A finite element model of the mandible was developed based on the information on its surface geometry obtained by three-dimensional laser scanning. The data point cloud was processed to produce a solid model in computer-aided design format. This solid model was then imported into a standard finite element analysis package and transformed into a three dimensional mesh. Two geometrically identical finite element models were created utilizing different algorithms of assigning mechanical properties of the bone. However, only one of them was employed in the present study due to insufficient data on the correlation of density and elastic modulus for right whale cortical bone.

Numerical simulations of bending with various choices of material parameters were conducted and compared with the full scale mechanical testing results. It was determined that the value $E_{C}=7.99 \mathrm{GPa}$ of Young's modulus for cortical bone provides the best overall correspondence of numerical and experimental results for longitudinal strain. Thus, this value is recommended for the overall vessel-whale collision model. Numerical modeling has also shown that predicted deformations are not sensitive to the choice of Poisson's ration for cortical bone. Note that parametric studies for material parameters of trabecular bone were not performed because these parameters were provided by the comprehensive mechanical testing reported in [9].

The finite element model utilized in this study assumes isotropic elastic behavior of cortical and trabecular bone tissue. However, experimental evidences suggest that both bone materials in the right whale mandible are anisotropic and inhomogeneous. For trabecular bone, this was shown explicitly by mechanical tests on the millimeter-scale specimens [9]. For cortical bone, the obvious evidence is the discrepancy between FEA-predicted and experimentally-observed transverse strains for the established equivalent value of $E_{c}$. One of the directions for future work planned by the authors is to collect more experimental data and establish correlation between anisotropic elastic parameters and tissue density of right whale mandible. Such information is not currently available so that it would not only provide better input for numerical modeling but be of a more general value for marine mammal biomechanics. 


\section{Acknowledgements}

The authors would like to acknowledge former UNH students Alexander Unrein, Matthew Packard and Robert Marsella who participated in the mechanical testing of the right whale mandible as part of their senior year capstone project. We are thankful to John Muller and Brian Snyder of Beth Israel Deaconess Medical Center for the bone material testing, and Lorna Gibson of MIT for her insightful comments on mechanical behavior of trabecular and cortical bones. Funding for this work was provided by the National Science Foundation (Graduate Research Fellowship Program, Campbell-Malone), the National Oceanic and Atmospheric Administration (Right Whale Grants Program, award number NA04NMF4720402), and the Woods Hole Oceanographic Institution Ocean Life Institute. All right whale samples were collected under National Marine Fisheries Service No. 932-1489-05.

\section{References}

[1] International Whaling Commission, Report of the Workshop on Status and Trends of Western North Atlantic Right Whales, 2001, Journal of Cetacean Research and Management, Special Issue 2, pp. 61-87.

[2] Campbell-Malone, R., Barco, S. G., Daoust, P.-Y., Knowlton, A. R., McLellan, W. A., Rotstein, D. S. and Moore, M. J.., 2008, "Gross and Histologic Evidence of Sharp and Blunt Trauma in North Atlantic Right Whales (Eubalaena glacialis) Killed by Vessels,” Journal of Zoo and Wildlife Medicine, 39(1), pp. 37-55.

[3] Moore, M.J., Knowlton, A.R., Kraus, S.D., McLellan, W.A., Bonde, R.K., 2005, "Morphometry, gross morphology and available histopathology in North Atlantic right whale (Eubalaena glacialis) mortalities (1970 to 2002),” Journal of Cetacean Research and Management 6 (3), pp. 199-214.

[4] Knowlton, A. R., Korsmeyer, F.T., Hynes, B., 1998, “The Hydrodynamic Effects of Large Vessels on Right Whales: Phase Two,” Final Report to the National Marine Fisheries Service, Northeast Fisheries Science Center, Woods Hole, MA.

[5] Laist, D.W., Knowlton, A.R., Mead, J.G., Collet, A.S., Podesta, M., 2001, “Collisions between ships and whales,” Marine Mammal Science 17(1), pp. 35-75.

[6] Vanderlaan, A.S.M., Taggart, C.T., 2007, "Vessel Collisions with Whales: The Probability of Lethal Injury Based on Vessel Speed,” Marine Mammal Science, 23(1), pp. 144-156.

[7] Jensen, A.S., and Silber, G. K., 2003, “Large whale ship strike database,” U.S. Department of Commerce, NOAA Technical Memorandum NMFS-F/OPR 25. 37 pp.

[8] National Marine Fisheries Service (NMFS)- National Oceanic and Atmospheric Administration (NOAA), 2008, "Endangered Fish and Wildlife; Final Rule to Implement Speed Restrictions to Reduce the Threat of Ship Collisions with North Atlantic Right Whales,” 
Department of Commerce Vol. 73, No. 198, 50 CFR Part 224 [Docket No. 040506143-7024-03], October 10, 2008.

[9] Campbell-Malone, R., 2007, "Biomechanics of North Atlantic Right Whale Bone: Mandibular Fracture as a Fatal Endpoint for Blunt Vessel-Whale Collision Modeling,” Ph.D. dissertation, Woods Hole Oceanographic Institution, Woods Hole, MA.

[10] Doblare, M., Garcia, J.M., Gomez, M.J., 2004, "Modelling Bone Tissue Fracture and Healing: A Review,” Eng. Fracture Mech., 71, pp. 1809-1840.

[11] Raymond, J. R., 2007, "Development of a Numerical Model to Predict Impact Forces in a North Atlantic Right Whale During Collision with a Vessel," M.S. thesis, University of New Hampshire, Durham, NH

[12] Campbell-Malone, R., M.J. Moore, J.A. Muller, and I. Tsukrov, 2008, “Material Properties of the North Atlantic Right Whale Mandible - Critical Data for Modeling Jawbone Fracture as Fatal Trauma Resulting from Vessel-Whale Collisions,” in prep., 39 pages.

[13] Right Whale Consortium, 2007, "North Atlantic Right Whale Consortium Photo-ID, Sightings, Genetics, Contaminants and Necropsy Database,” New England Aquarium, Boston, MA, USA.

[14] Bathe, K-J, 1996, “Finite Element Procedures,” Prentice-Hall, Inc., Upper Saddle River, NJ, USA.

[15] Yosibash, Z., Trabelsi, N., Milgrom, C., 2007, "Reliable Simulations of the Human, Proximal Femur by High-Order Finite Element Analysis Validated by Experimental Observations,” J. Biomechanics, 40, pp. 3688-3699.

[16] Song, Y., Debski, R. E., Musahl, V., Thomas, M., and Woo, S.L.-Y., 2004, “A ThreeDimensional Finite Element Model of the Human Anterior Cruciate Ligament: a Computational Analysis with Experimental Validation,” J. Biomechanics, 37, pp. 383-390

[17] Taddeia, F., Cristofolinia, L., Martellia, S., Gillc, H.S., and Viceconti, M., 2006, "SubjectSpecific Finite Element Models of Long Bones: an In Vitro Evaluation of the Overall Accuracy,“ J. Biomechanics. 39, pp. 2457-2467

[18] Reina, J.M., Garcia-Aznar, J.M., Dominguez, J., Doblare, M., 2007, "Numerical Estimation of Bone Density and Elastic Constants Distribution in a Human Mandible,” J. Biomechanics, 40, pp. 828-836.

[19] Ashman, R.B., Cowin, R.B., Van Buskirk, W.C., Rice, J.C., 1984, “A Continuous Wave Technique for the Measurement of the Elastic Properties of Cortical Bone,” J. Biomechanics, 17, pp. 349-361. 
[20] Ashman, R.B., Van Buskirk, W.C., 1987, “The Elastic Properties of a Human Mandible,” Advances in Dental Research, 1, pp. 64-67.

[21] Misch, C. E., Z. Qu and M. W. Bidez, 1999. "Mechanical Properties of Trabecular Bone in the Human Mandible: Implications for Dental Implant Treatment Planning and Surgical Placement,” Journal of Oral and Maxillofacial Surgery, 57 (6), pp. 700-706.

[22] Giesen, E., M. Ding, M. Dalstra and van Eijden, T., 2004. "Changed Morphology and Mechanical Properties of Cancellous Bone in the Mandibular Condyles of Edentate People," Journal of Dental Research, 83 (3), pp. 255-259.

[23] Galante, J., W. Rostoker and Ray, R. D., 1970, “Physical Properties of Trabecular Bone,” Calcified Tissue International, 5(1), pp. 236-246.

[24] Carter, D.R. and Hayes, W.C., 1977, "The Compressive Behavior of Bone as a Two-Phase Porous Structure,” The Journal of Bone and Joint Surgery, 59(7), pp. 954-962.

[25] Gibson, L. J. and Ashby, M. F., 1997, Cellular Solids: Structure and Properties, Cambridge University Press, Cambridge, UK, Chap. 11, pp. 429-452.

[26] Keller, T. S., 1994, “Predicting the Compressive Mechanical Behavior of Bone,” Journal of Biomechanics 27(9), pp. 1159-1168.

[27] Hong, J., Cabe, G. D., Tedrow, J. R., Hipp, J. A., and Snyder, B. D., 2004, “Failure of Trabecular Bone with Simulated Lytic Defects Can Be Predicted Non-Invasively by Structural Analysis,” Journal of Orthopaedic Research, 22, pp. 479-486.

[28] Tsukrov, I., Baldwin, K., DeCew, J., and Raymond, J., 2007, “Finite Element Modeling of a Right Whale Mandible Strength and Ship-Whale Collision Dynamics,” Final Report to Woods Hole Oceanographic Institution, University of New Hampshire, Durham, NH.

[29] Reilly, D.T., and Burstein, A.H., 1975, "The Elastic and Ultimate Properties of Compact Bone Tissue,” J. Biomechanics, 8, pp. 393-405.

[30] Pithioux, M., Lasaygues, P., and Chabrand, P., 2002, “An Alternative Method to Determine The Elastic Properties of Cortical Bone,” J. Biomechanics, 35, pp. 961-968.

[31] Shahar, R., Zaslavsky, P., Barak, M., Friesem, A.A., Currey J.D., and Weiner, S., 2007, "Anisotropic Poisson's Ratio and Compression Modulus of Cortical Bone Determined by Speckle Interferometry,” J. Biomechanics, 40, pp. 252-264.

[32] Chen, P.H., 1978, "Finite Element Dynamic Structural Model of the Human Thorax for Chest Impact Response and Injury Studies,” Aviat. Space Environ. Med., 49, pp. 143-149.

[33] Niu, Y., Shen, W., Stuhmiller, J.H., 2007, "Finite Element Models of Rib as an Inhomogeneous Beam Structure Under High-Speed Impacts,” Med. Eng. Phys., 29, pp. 788-798. 
[34] Currey, J.D., 2004, “Tensile Yield in Compact Bone Is Determined by Strain, Post-Yield Behaviour by Mineral Content,” J. Biomechanics, 37, pp. 549-556. 